\title{
Real-time-dynamics quantum simulation of (1+1)-dimensional lattice QED with Rydberg atoms
}

\author{
Simone Notarnicola $\odot,{ }^{1,2, *}$ Mario Collura, ${ }^{1,3}$ and Simone Montangero ${ }^{1,2}$ \\ ${ }^{1}$ Dipartimento di Fisica e Astronomia “G. Galilei”, via Marzolo 8, I-35131 Padova, Italy \\ ${ }^{2}$ INFN, Sezione di Padova, via Marzolo 8, I-35131 Padova, Italy \\ ${ }^{3}$ SISSA, Via Bonomea 265, 34136 Trieste, Italy
}

(Received 23 August 2019; revised manuscript received 19 December 2019; accepted 17 January 2020; published 10 March 2020)

\begin{abstract}
We show how to implement a Rydberg-atom quantum simulator to study the nonequilibrium dynamics of an Abelian (1+1)-dimensional lattice gauge theory. The implementation locally codifies the degrees of freedom of a $\mathbf{Z}_{3}$ gauge field, once the matter field is integrated out by means of the Gauss local symmetries. The quantum simulator scheme is based on currently available technology and thus is scalable to considerable lattice sizes. It allows, within experimentally reachable regimes, us to explore different string dynamics and to infer information about the Schwinger U(1) model.
\end{abstract}

DOI: 10.1103/PhysRevResearch.2.013288

\section{INTRODUCTION}

Rydberg-atom systems are nowadays one of the most promising and versatile platforms in the field of quantum simulation for the achievement of results inaccessible via classical numerical simulations [1-7]. The internal ground state of neutral atoms is coupled to a highly excited Rydberg state, realizing a benchmarked qubit prototype [8-11]. The strong dipole-dipole coupling between excited atoms induces the Rydberg blockade mechanism [12], allowing one to engineer local dynamical constraints. Optical tweezers arrays allow one to trap and arrange a large number of atoms in various geometries, from one-dimensional lattices to unconventional three-dimensional structures $[2,13]$. Recent experimental results concerning a Rydberg-atom chain have raised interest in studying possible connections between Abelian lattice gauge theories and Rydberg-atom systems [2,14]. Despite the proposals realized in the past decade for studying Abelian and non-Abelian lattice gauge theories via universal quantum simulators [15-28], the search for a mapping suitable for systems with large lattice sizes is ongoing. In recent experiments, trapped ions [29] and ultracold atoms [30,31] have been used to explore the mechanism of pairs of particle-antiparticle production in the Schwinger model for minimal-size systems, while hybrid protocols combining quantum simulation [32] or computation [33] with classical numerical simulations have been set up. The realization of a quantum simulator scalable to large lattice sizes, even for minimal gauge models such as the Abelian ones, would pave the way to study high-energy physics nonlocal phenomena, such as scattering processes and string breaking [34,35], providing motivation for our work.

\footnotetext{
*Corresponding author: simone.notarnicola@gmail.com

Published by the American Physical Society under the terms of the Creative Commons Attribution 4.0 International license. Further distribution of this work must maintain attribution to the author(s) and the published article's title, journal citation, and DOI.
}

We propose a Rydberg-atom quantum simulator to study a one-dimensional (1D) Abelian lattice gauge theory with spinless fermions coupled to an electric field. Fermions exist on the lattice sites while the electric field is defined on each link between two neighboring sites. In particular, we map the dynamics of our simulator into the gauge-invariant dynamics of the electric field. We focus on an electric field string generated by two opposite charges separated on the lattice to study different dynamical regimes: The string can persist in time or it can be broken by the spontaneous creation of particle-antiparticle pairs in the middle of it, in analogy with the confinement properties of QCD [18,36,37].

Encoding a quantum gauge field into atomic degrees of freedom imposes the discretization and truncation of its spectrum. Starting from the Schwinger model, we replace the continuous symmetry group $\mathrm{U}(1)$ with $\mathbf{Z}_{n}$, in which the electric field spectrum contains $n$ discrete values. We choose $n=3$ so that the spectrum of the local electric field is $(0, \pm 1)$ [Fig. 1(a)].

Differently from the well-studied case of $n=2$ [14,38], in which the spectrum is $( \pm 1 / 2)$, in our case the electric field energy contribution is nontrivial [see Eq. (3)]. We can therefore induce different string behaviors by changing the values of the couplings. The $\mathbf{Z}_{3}$ gauge symmetry is encoded in the lattice geometry of our quantum simulator, as shown in Fig. 1(b). Groups of three Rydberg atoms represent each link, and we map the eigenstates of the local electric field into the configurations with only one atom excited [Fig. 1(c)]. By applying an effective Rabi frequency $\Omega$, we allow each atom to oscillate between the ground state and the Rydberg state. By tuning their interatomic distances, we exploit the Rydberg blockade to suppress those atomic configurations corresponding to states which break the Gauss law in a given charge sector. As a result, we find that the dynamics of the atomic excitations reproduces the gauge-invariant dynamics of the $\mathbf{Z}_{3}$ gauge field. The Hamiltonian parameters can be modulated by changing a local detuning applied to each atom [Fig. 1(c)]. 


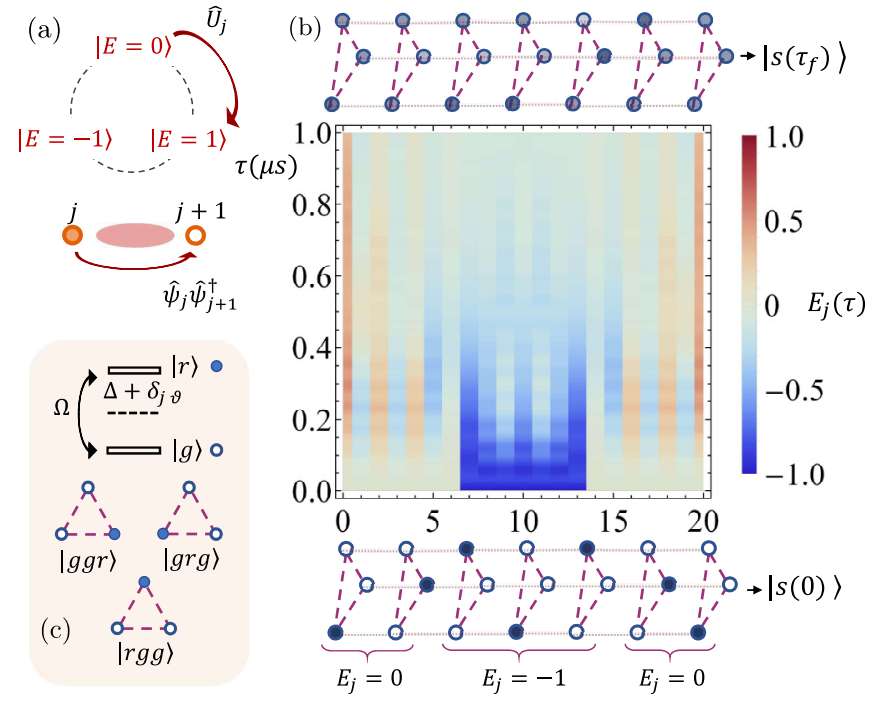

FIG. 1. (a) Dynamics of the $\mathbf{Z}_{3}$ gauge and fermion field relative to the Hamiltonian $\hat{H}$ of Eq. (3): A rotation of the local gauge field state is accompanied by a fermion hopping process which preserves gauge invariance. Given a set of three equidistant atoms, the electric field eigenstates $|E\rangle_{j}$ are mapped into those configurations with only one excited atom. (b) Chain of atomic triangular sets. An electric field string is represented by the separable state $|s(0)\rangle$ at $\tau=0$ : By setting the parameters in the Hamiltonian $\hat{H}_{r}$ [see Eq. (4)] the system evolves to the state $\left|s\left(\tau_{f}\right)\right\rangle$ in which the initial string is broken. (c) The ground state of the atoms (empty dot) is coupled to a Rydberg state (full dot) by a Rabi frequency $\Omega$ with a local blue-shifted detuning $\Delta+\delta_{j \vartheta}$. Numerical parameters of Hamiltonian in Eq. (3): $m=0, t=0.682 \mathrm{MHz}, g^{2} / t=0.5$.

We check the reliability of our quantum simulator in two steps. First, we compare the $\mathbf{Z}_{3}$ and U(1) models, discussing under what conditions their dynamics coincide. Despite some differences, we find that the parameter regime wherein stringbreaking dynamics occurs is the same for both theories. Second, we compare the dynamics of the $\mathbf{Z}_{3}$ model with that of our quantum simulator, showing that our scheme allows us to investigate the two different string dynamics regimes. We conclude that our quantum simulator is able to capture reliable features of continuous and discrete Abelian lattice gauge theories.

\section{MODEL}

The Hamiltonian for the $(1+1)$-dimensional lattice QED in the Schwinger formulation is $[39,40]$

$$
\hat{H}_{S}=\sum_{j}\left[-t \hat{\psi}_{j}^{\dagger} \hat{\mathcal{U}}_{j} \hat{\psi}_{j+1}+\text { H.c. }+m p_{j} \hat{n}_{j}+g^{2} \hat{\mathcal{E}}_{j}^{2}\right] \text {, }
$$

where a staggered, fermionic, spinless matter field with mass $m$ is defined on the lattice sites. It satisfies $\left\{\hat{\psi}_{j}^{\dagger}, \hat{\psi}_{k}\right\}=\delta_{j k}$ and $\left\{\hat{\psi}_{j}, \hat{\psi}_{k}\right\}=\left\{\hat{\psi}_{j}^{\dagger}, \hat{\psi}_{k}^{\dagger}\right\}=0$, while $\hat{n}_{j}=\hat{\psi}_{j}^{\dagger} \hat{\psi}_{j}$ and $p_{x}=(-1)^{j}$. The gauge field propagator $\hat{\mathcal{U}}_{j}$ and the electric field $\hat{\mathcal{E}}_{j}$ are defined on each link between the nearest-neighbor sites $j$ and $j+1$ : They commute according to $\left[\hat{\mathcal{E}}_{j}, \hat{\mathcal{U}}_{k}\right]=\delta_{j k} \hat{\mathcal{U}}_{j}$ and $g^{2}$ is the electric field energy coupling.

Due to the staggering, the electrons (positrons) are represented by filled (empty) even (odd) sites and therefore the gauge-matter interaction term proportional to $t$ is responsible for electron-positron pair creation and annihilation. During these processes, the electric field is incremental or decremental in order to satisfy the Gauss law on each site: Equivalently, given the set of gauge operators $\hat{Q}_{j}=\Delta \hat{\mathcal{E}}_{j}-\frac{1-p_{j}}{2}+e \hat{n}_{j}$, with $\Delta \hat{\mathcal{E}}_{j}=\hat{\mathcal{E}}_{j}-\hat{\mathcal{E}}_{j-1}$ and $e=-1$, we have $\left[\hat{H}_{S}, \hat{Q}_{j}\right]=0 \forall j$.

It follows that the Hamiltonian has a block-diagonal form in the basis of the gauge operators' eigenstates. Each block is identified by a set of static charges $g_{j}$ and the relative states satisfy $\hat{Q}_{j}|\psi\rangle=g_{j}|\psi\rangle \forall j$.

Once the boundary conditions and the set of static charges $g_{j}$ are fixed, the gauge operators $\hat{Q}_{j}$ fix a one-to-one correspondence between the eigenstates of the matter and the electric field operators $\hat{\psi}_{j}^{\dagger} \hat{\psi}_{j}$ and $\hat{\mathcal{E}}_{j}$, indicated as $\{|m\rangle\}$ and $\{|\mathcal{E}\rangle\}$, respectively. Therefore, the basis of the gauge sector characterized by $\left\{g_{j}\right\}$ is in the form $\left|m, \mathcal{E} ;\left\{g_{j}\right\}\right\rangle$. It follows that $\hat{H}_{S}$ can be recast in each sector as a function of the matter or the gauge field operators $[41,42]$. Hereafter, we set $g_{j}=0 \forall j$ and write the Hamiltonian as a function of the gauge field operators. The Hamiltonian is still local, namely (see Appendix A),

$$
\begin{aligned}
\hat{H}_{S}^{g}= & -t \sum_{j} \hat{\mathcal{U}}_{j}^{\dagger} \hat{P}_{\Delta \mathcal{E}_{j}}(0) \hat{P}_{\Delta \mathcal{E}_{j+1}}(1)+\text { H.c. } \\
& +m \sum_{j}\left(\frac{1-p_{j}}{2}-\Delta \hat{\mathcal{E}}_{j}\right)+g^{2} \sum_{j} \hat{\mathcal{E}}_{j}^{2} .
\end{aligned}
$$

The projectors $\hat{P}_{\Delta \mathcal{E}_{j}}\left(n_{j}\right)$ select the electric field configurations whose expectation values satisfy the local gauge-invariance condition $\Delta \mathcal{E}_{j}=\frac{1-p_{j}}{2}+n_{j}$. As a consequence, the hopping matrix elements of the Hamiltonian $\hat{H}_{S}$ between the states $|m, \mathcal{E}\rangle$ and $\left|m^{\prime}, \mathcal{E}^{\prime}\right\rangle$ coincide with those of $\hat{H}_{S}^{g}$ computed over the states $|\mathcal{E}\rangle$ and $\left|\mathcal{E}^{\prime}\right\rangle$.

In order to encode the gauge degrees of freedom in a quantum simulator, we need to truncate and discretize the spectrum of the electric field. To this purpose, we replace the continuous-spectrum operator $\hat{\mathcal{U}}_{j}$ with the discrete clock operator $\hat{U}_{j}$ such that $\hat{U}_{j}^{n}=\left(\hat{U}_{j}^{\dagger}\right)^{n}=\mathbf{1}$ with $n \in \mathbb{N}$, that is, we move from the continuous gauge symmetry group $\mathrm{U}(1)$ to $\mathbf{Z}_{n}[43,44]$. We set $n=3$, so the electric field $\hat{E}_{j}$ admits only three possible states $\{|-1\rangle,|0\rangle,|+1\rangle\}$ and the operators $\hat{U}_{j}\left(\hat{U}_{j}^{\dagger}\right)$ cyclically permute them clockwise (counterclockwise) as shown in Fig. 1(a). Gauge invariance is guaranteed by the condition $\tilde{\Delta} \hat{E}_{j}=\left(\frac{1-p_{j}}{2}+\hat{n}_{j}\right) \bmod 3$ with the spectrum $\{-1,0,1\}$. Finally, we define the electric field energy to be proportional to $\sum_{j} \hat{E}_{j}^{2}$. In conclusion, the Hamiltonian reduces to

$$
\begin{aligned}
\hat{H}= & -t \sum_{j} \hat{U}_{j}^{\dagger} \hat{P}_{\tilde{\Delta} E_{j}}(0) \hat{P}_{\tilde{\Delta} E_{j+1}}(1)+\text { H.c. } \\
& +m \sum_{j}\left(\frac{1-p_{j}}{2}-\tilde{\Delta} \hat{E}_{j}\right)+g^{2} \sum_{j} \hat{E}_{j}^{2} .
\end{aligned}
$$




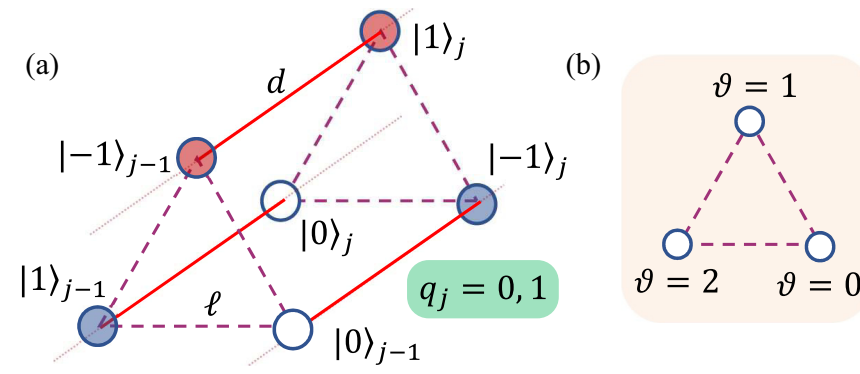

FIG. 2. Atomic lattice geometry. (a) The distances $\ell$ and $d$ are short enough such that Rydberg blockade prevents simultaneous excitations of atoms in the same link or in different links but aligned along the lattice axes (red filled circles). Simultaneous excitations of nonaligned atoms from different links are allowed (blue filled circles). (b) A label $\vartheta$ is assigned to the atoms, uniformly for each link, to map each $|E\rangle_{j}$ into the states $|\vartheta\rangle_{j}$.

\section{RYDBERG QUANTUM SIMULATOR}

Our quantum simulator consists of a quasi-1D lattice of neutral atoms coupled to a Rydberg state $n S$, with $n \gg 1$, by an effective Rabi frequency $\Omega$. The atoms are initially trapped into a tweezers array $[13,45,46]$ and then released. By locally modulating the laser detuning, a configuration in which some atoms are excited to a Rydberg state and the others are in their internal ground state is created. A nontrivial dynamics is then induced by remodulating the laser detuning: The atoms move from ground to Rydberg states and thus interact among each other. In the following we show that this process effectively reproduces the gauge-invariant dynamics of the electric field in the $\mathbf{Z}_{3}$ model. We start by showing that the gauge-invariant electric field eigenstates are mapped into a set of atomic configurations in which the atoms are in their ground or Rydberg states.

In general, two atoms separated by a distance $r$ can be simultaneously excited only if $\Omega>V_{r}=c_{6} / r^{6}$, where $V_{r}$ is the van der Waals interaction energy, due to the so-called Rydberg blockade mechanism, and $c_{6}$ depends on the atomic species and on the specific excited state [12]. We impose gauge invariance by mapping gauge-breaking states into atomic configurations forbidden by the Rydberg blockade.

The lattice is shaped as a prism with an equilateral triangular section, as shown in Fig. 2(a). Sets of three atoms, called links, lay in planes perpendicular to the main prism axes. The links are placed at a fixed distance $d$ from each other, while the distance between atoms in the same set is $\ell$. Their dynamics is described by the Hamiltonian $[2,3,47]$

$$
\hat{H}_{r}=\sum_{j, \vartheta}\left[\Omega \hat{\sigma}_{j \vartheta}^{x}-\Delta_{j}^{\vartheta} \hat{n}_{j}^{\vartheta}+\frac{1}{2} \sum_{\vartheta^{\prime}, k} V_{j, k}^{\vartheta, \vartheta^{\prime}} \hat{n}_{j}^{\vartheta} \hat{n}_{k}^{\vartheta^{\prime}}\right],
$$

where each atom is labeled by the link index $j$ or $\vartheta$, which indicates the position inside the link [Fig. 2(b)]. The ground state of each atom $|g\rangle_{j \vartheta}$ is coupled to the excited state $|r\rangle_{j \vartheta}$ by the operator $\sigma_{j \vartheta}^{x}$ with Rabi frequency $\Omega$. The projector $\hat{n}_{j \vartheta}=|r\rangle\left\langle\left. r\right|_{j \vartheta}\right.$ is multiplied by a detuning term with $\Delta_{j}^{\vartheta}=$ $\Delta-\delta_{j \vartheta}>0$ and $\delta_{j \vartheta} \ll \Delta$. The interaction depends only on the distance between the atoms. We define $V_{\ell}=V_{j, j}^{\vartheta, \vartheta^{\prime}}$ and
$V=V_{j, j+1}^{\vartheta, \vartheta^{\prime}}$ with $\vartheta \neq \vartheta^{\prime}$ and $V_{d}=V_{j, j+1}^{\vartheta, \vartheta}$. We neglect $V_{j, k}^{\vartheta, \vartheta^{\prime}}$ for $|k-j|>1$ since they are much smaller than the other energies involved.

We first map the gauge-invariant states into a set of atomic configurations and then we map the Hamiltonian $\hat{H}$ into $\hat{H}_{r}$. We choose $\Delta \gg \Omega$ and $V_{\ell} \gg \Omega$. For each single link, Rydberg excitations are enhanced by the large detuning, but simultaneous excitations are prevented by Rydberg blockade. By applying second-order perturbation theory we restrict the dynamics of the $j$ th link to the subspace spanned by a set of states $\Sigma_{j}=\left\{|\vartheta\rangle_{j}\right\}_{0 \leqslant \vartheta \leqslant 2}$ in which the atom in position $\vartheta$ is excited [Fig. 1(a)].

We now consider a chain of $L$ links, arranged as shown in Fig. 2, with the distance $d$ between consecutive links such that $V_{d} \gg \Omega$ and $V \sim \Omega$. Simultaneous excitations of aligned atoms (red filled circles) are forbidden, while nonaligned excitations (blue filled circles) are allowed. We map in a staggered fashion the electric field eigenstates $|E\rangle_{j}$ into the states $|\vartheta\rangle_{j}$ according to $\vartheta_{j}=\left[-E_{j}+4+(-1)^{j}\right] \bmod 3$. The set of allowed atomic configurations $\Phi \subset \bigotimes_{j=1}^{L} \Sigma_{j}$ corresponds to the set of $\mathbf{Z}_{3}$ gauge-invariant states. In Fig. 2(a) we show two neighboring links and the site in between with charge $q_{j}=0$, +1 . Since the electric field cannot decrease from the link $j-1$ to the link $j$, electric field states such as $|0\rangle_{j-1}|-1\rangle_{j}$ are mapped into configurations of excited atoms prevented by Rydberg blockade. On the other side, the configurations corresponding to the states $|0\rangle_{j-1}|0\rangle_{j}\left(q_{j}=0\right)$ or $|0\rangle_{j-1}|1\rangle_{j}$ $\left(q_{j}=1\right)$ are allowed.

In order to map the Hamiltonian $\hat{H}$ [Eq. (3)] into $\hat{H}_{r}$ defined in Eq. (4) we must confine the dynamics into the subspace spanned by $\Phi$. By applying second-order perturbation theory we obtain the effective Hamiltonian $\hat{H}_{r}^{\Phi}=-t \sum_{j, \vartheta \neq \vartheta^{\prime}}^{\prime}|\vartheta\rangle\left\langle\left.\vartheta^{\prime}\right|_{j}+\sum_{j, \vartheta} \delta_{j, \vartheta} \mid \vartheta\right\rangle\left\langle\left.\vartheta\right|_{j}\right.$, where $t=$ $\Omega^{2}\left[1 /(\Delta-2 V)+1 /\left(V_{l}+2 V-\Delta\right)\right]$ and the energy shift $L \Delta-(L-1) V$ has been applied (see Appendix B). The primed sum is restricted to the transitions between states in $\operatorname{span}(\Phi)$ and is equivalent to the hopping term of the Hamiltonian $\hat{H}$ by construction. The electric field energy coupling $g^{2}$ is obtained by modulating the local detuning $\delta_{j, \vartheta}$ such that $g^{2}=\delta_{j, \vartheta^{\prime}}-\delta_{j, \vartheta}$, with $\left|\vartheta^{\prime}\right\rangle \equiv|E= \pm 1\rangle$ and $|\vartheta\rangle \equiv|E=0\rangle$.

The mass term involves an interaction between nearestneighbor links in the Hamiltonian $\hat{H}$. Its implementation should be encoded in the interlink interaction term of the Hamiltonian $\hat{H}_{r}$. The Hamiltonian $\hat{H}_{r}^{\Phi}$ implements the case with $m=0$. Indeed, two-link states with or without charge are both represented by configurations whose interaction energy is $V$. In Appendix $C$ we show that the case $m \neq 0$ can be implemented by modifying the geometry of the lattice.

\section{THE $Z_{3}$ MODEL DYNAMICS}

The agreement between the dynamics of the $\mathbf{Z}_{3}$ and Schwinger models depends on the parameter $\Gamma=g^{2} / t$. In the limit $\Gamma>1$ the $\mathbf{Z}_{3}$ model better approximates the Schwinger one: Its dynamics is naturally constrained in the low-energy sector due to the large electric field coupling and it is not affected by the truncation of the electric field spectrum. As an example, we compare the dynamics of the two models by taking the bare vacuum, with $E_{j}=0 \forall j$, as the initial state. 


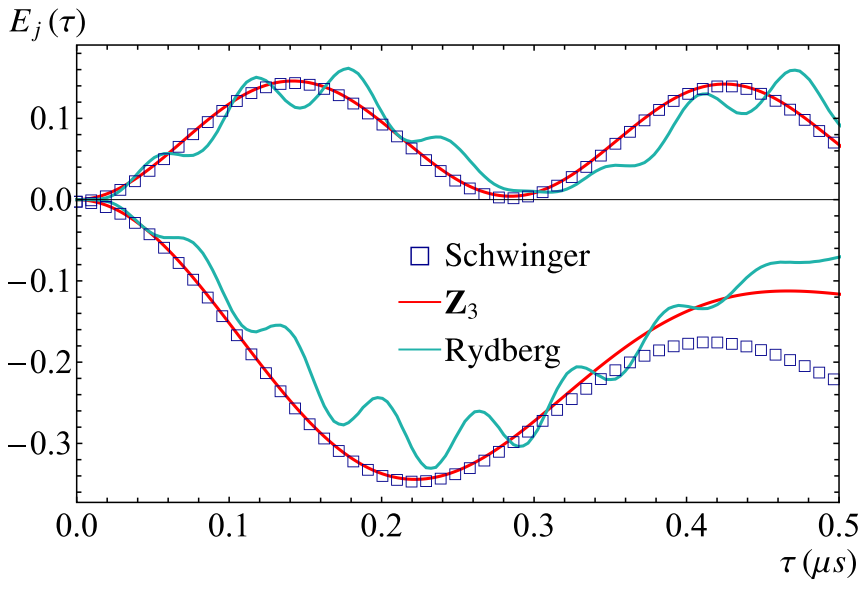

FIG. 3. Electric field bulk local dynamics. The dynamics of the quantum simulator (teal curve) and the $\mathbf{Z}_{3}$ model (red curve) exhibit fair agreement for $\Gamma=4.0$ (upper curves, $j=10$ ) and $\Gamma=0.5$ (lower curves, $j=11$ ). In the first case they coincide also with the dynamics of the Schwinger model (blue squares), as expected in the limit $\Gamma \gg 1$. In the latter they deviate due to the truncation of the electric field spectrum in the $\mathbf{Z}_{3}$ model. The parameters are $L=21, t=0.682 \mathrm{MHz}$, and $m=0$.

In Fig. 3 (upper panel) we show the local bulk dynamics of the electric field for $\Gamma=4$. The $\mathbf{Z}_{3}$ dynamics (red curve) and the Schwinger dynamics (squares) coincide. In the opposite regime, with $\Gamma=0.5$ the dynamics of the two models relax to different values (bottom panel of Fig. 3).

In order to investigate the string dynamics, we take as the initial state $|s(0)\rangle$ an electric field string of length $s$ originated by a positron-electron pair. The state evolves as $|s(\tau)\rangle=$ $\exp \{-i \hat{H} \tau\}|s(0)\rangle(\hbar=1) .{ }^{1}$ Although the ground state of the Hamiltonian $\hat{H}$ is always confined, the unitary dynamics induced by quenching $\Gamma$ allows us to observe different regimes [36].

For $\Gamma \lesssim 1$ fluctuations of local charge density lead to the creation of positron-electron pairs which annihilate the electric field between them and break the string. We show an example of this dynamics in Fig. 4(a), where a chain of $L=21$ links has been prepared with an electron-positron string of length $s=7$. By quenching $\Gamma$ from $\Gamma=0$ to $\Gamma=0.5$ the string breaks during its evolution. A different scenario emerges by choosing instead $\Gamma>1$. In Fig. 4(c) we take the same initial state and evolve it with $\Gamma=4.0$. Due to the large gap between the zero and nonzero electric field states, the string-breaking process is strongly off-resonant and does not occur in accessible times. It is worth noting the oscillations in the middle of the string, which are a peculiarity of the $\mathbf{Z}_{3}$ model: The electric field is oscillating between the two local degenerate states $|E= \pm 1\rangle_{j}$ and the transient in which $\left\langle\hat{E}_{j}\right\rangle=0$ is due to their superposition during the population inversion process.

\footnotetext{
${ }^{1}$ The dynamics of both $\mathbf{Z}_{3}$ and $U(1)$ models have been obtained by exact diagonalization.
}

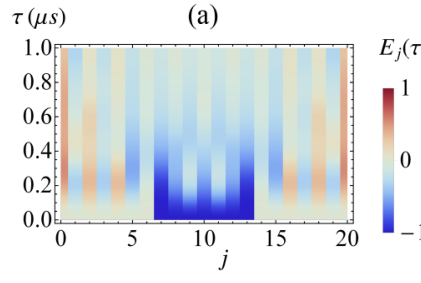

$\tau(\mu s)$
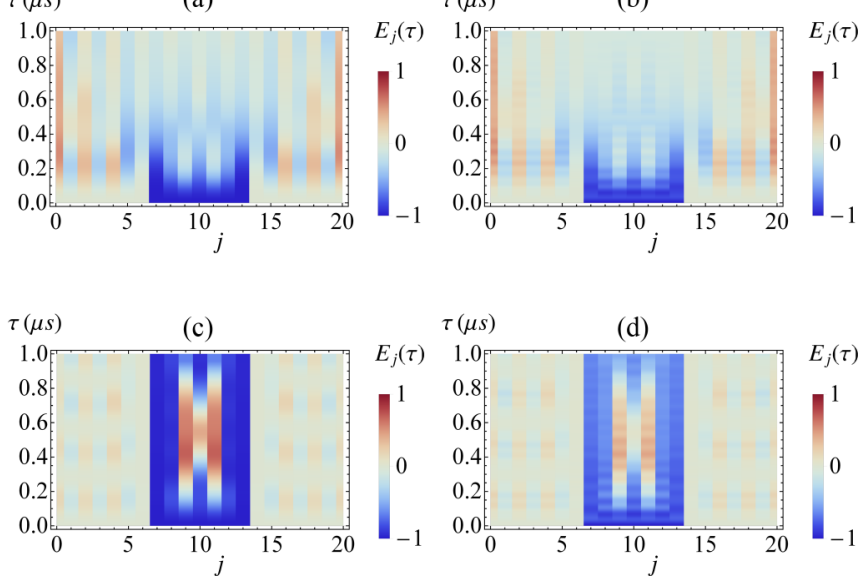

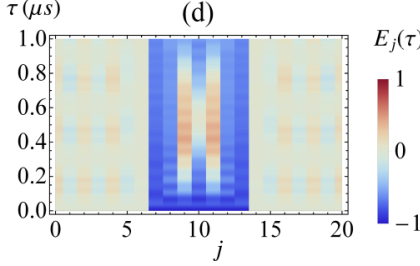

FIG. 4. (a) and (c) String dynamics of the $\mathbf{Z}_{3}$ model. (b) and (d) Rydberg-atom simulator dynamics. For $\Gamma=0.5$ (upper line) the initial string breaks; for $\Gamma=4.0$ (bottom line) the string is not broken during time evolution. The oscillation of the electric field expectation value inside the string is not due to string breaking but to the intrinsic dynamics of the $\mathbf{Z}_{3}$ model. The parameters are $L=21$, $t=0.682 \mathrm{MHz}$, and $m=0$.

\section{RESULTS}

We benchmark the dynamics of the quantum simulator via a numerical analysis. The experimental parameters we use refer to ${ }^{87} \mathrm{Rb}$ atoms excited to the state $|68 S ; m=1 / 2\rangle$, with $c_{6}=612 \mathrm{GHz}$. We set $\Omega=3 \mathrm{MHz}, \Delta=27 \mathrm{MHz}$, $\ell=4 \mu \mathrm{m}$, and $d=5.5 \mu \mathrm{m}$ so that we have $t=0.682 \mathrm{MHz}$. We set $\delta_{j, \vartheta}=0$ for $\vartheta, j$ such that $E_{j}=0$ and $\delta_{j, \vartheta}=g^{2}$ for $\vartheta, j$ such that $E_{j}= \pm 1$. By calling $V^{(2)}$ the amplitude of next-nearest-neighbor interactions, we have $V^{(2)} \ll V, V_{\ell}, V_{d}$ by construction. The values we choose for the parameters of the Hamiltonian $\hat{H}_{r}$ guarantee that $V^{(2)}<t, g^{2}$. We have numerically checked that, under these conditions, the dynamics of the $\mathbf{Z}_{3}$ model is not significantly affected by next-nearestneighbor interactions and therefore we neglect them. ${ }^{2}$ We use a time-dependent block decimation (TEBD) algorithm $[48,49]$ to simulate the dynamics of the Rydberg Hamiltonian $\hat{H}_{r}$ (see Appendix D) and compare it with the exact diagonalization of the $\mathbf{Z}_{3}$ model for a chain of $L=21$ links. The implementation thus requires 60 atoms and is achievable on the basis of a recent experiment [3]. In Fig. 3 we compare the dynamics of the local electric field obtained from the Rydberg quantum simulator against the exact $\mathbf{Z}_{3}$ one. The curves relative to the $\mathbf{Z}_{3}$ model (red line) and to the quantum simulator (teal line) are in a fair agreement in the cases with $\Gamma>1$ (upper panel) and $\Gamma<1$ (lower panel). The high-frequency oscillations in the curve relative to the Rydberg dynamics are reminiscent of the second-order processes in $\Omega$ which are generated in the transitions between different states $|\vartheta\rangle_{j}$.

\footnotetext{
${ }^{2}$ Next-nearest-neighbor interactions between excited atoms add an extra term in the Hamiltonian $\hat{H}_{r}^{\Phi}$ which does not break the gauge invariance, being diagonal in the electric field configurations basis. We have numerically checked that the dynamics generated by the Hamiltonians $\hat{H}_{r}^{\Phi}$, or equivalently $\hat{H}$, is not significantly affected by this term.
} 
This Rydberg-atom quantum simulator is able to catch the string dynamics. As we show in Figs. 4(b) and 4(d), it is possible to distinguish the string breaking and persisting regimes predicted by the $\mathbf{Z}_{3}$ model.

\section{CONCLUSION AND OUTLOOK}

In this work we have introduced a quantum simulator for the study of the real time dynamics of an Abelian quantum lattice gauge theory with scalable sizes of the lattice up to $\sim 20$ sites. Due to the Rydberg blockade mechanism, which is able to guarantee a high reliability between the original model and the experimental realization in the case of local interactions, we explored the dynamics of the $\mathbf{Z}_{3}$ gauge model in different parameter regimes and with different initial states. We compared the dynamics of the $\mathbf{Z}_{3}$ model with that of the $\mathrm{U}(1)$ gauge theory, finding remarkable similarities of the string dynamics. Our quantum simulator is therefore a versatile and reliable experimental setup useful for investigating exotic properties of discrete and continuous Abelian lattice gauge theories [50-52]. A possible outlook for this work is the extension to two-dimensional theories, in continuity with a recent proposal about the study of two-dimensional pure gauge systems [38], as well as the application of this protocol for simulating clock variables to different models such as time crystals $[53,54]$.

\section{ACKNOWLEDGMENTS}

We acknowledge insightful discussions with P. Zoller, M. Lukin, A. Celi, P. Silvi, and S. Pascazio. This work is partially supported by the Italian PRIN 2017, the Horizon 2020 research and innovation programme under Grant Agreement No. 817482 (PASQuanS), the QuantERA project QTFLAG and QuantHEP, the DFG via the TWITTER project. S.N. would like to thank the Erwin Schrödinger International Institute (ESI) in Wien for the hospitality and support during the Programme on "Quantum Simulation-from Theory to Applications". S.M. would like to thank the Kavli Institute for Theoretical Physics (KITP) in Santa Barbara for the support and hospitality during the QSIM19 program. The authors acknowledge support by the state of Baden-Württemberg through bwHPC and the German Research Foundation (DFG) through Grant No. INST 40/467-1 FUGG (JUSTUS cluster). The authors acknowledge the CINECA award under the ISCRA initiative, for the availability of high performance computing resources and support.

\section{APPENDIX A: INTEGRATING THE MATTER FIELD}

In this Appendix we derive the Hamiltonian $\hat{H}_{S}^{g}$ of Eq. (2) starting from $\hat{H}_{S}$ defined in Eq. (1). The subspace we are considering contains those states $|\Phi\rangle$ such that $\hat{Q}_{j}|\Phi\rangle=0 \forall j$. The basis vectors of this subspace, $\{|m, \mathcal{E}\rangle\}$, are the eigenstates of a projector operator $\hat{P}$ which can be written as a product of local terms $\hat{P}=\prod_{j=1}^{L} \hat{P}_{j}$. We express $\hat{P}_{j}$ in terms of the local computational basis basis $\prod_{j=1}^{L}\left\{|m\rangle_{j}\right\} \otimes\left\{|\hat{\mathcal{E}}\rangle_{j}\right\}$.

In particular, since the Gauss law establishes a relation between the expectation value of the charge density operator $\hat{n}_{j}$ and the related electric field flux $\Delta \hat{\mathcal{E}}_{j}=\hat{\mathcal{E}}_{j}-\hat{\mathcal{E}}_{j-1}$, we define the projectors

$$
\hat{P}_{\Delta \mathcal{E}_{j}}\left(n_{j}\right)=\sum_{\mathcal{E}}|\mathcal{E}\rangle\left\langle\left.\mathcal{E}\right|_{j-1} \otimes \mid \mathcal{E}+\Delta \mathcal{E}^{n_{j}}\right\rangle\left\langle\mathcal{E}+\left.\Delta \mathcal{E}^{n_{j}}\right|_{j},\right.
$$

with $\Delta \mathcal{E}^{n_{j}} \equiv \mathcal{E}_{j}-\mathcal{E}_{j-1}=\frac{1-p_{j}}{2}-n_{j}$. With this representation we have that

$$
\hat{P}_{j}=|0\rangle\left\langle\left. 0\right|_{j} \otimes \hat{P}_{\Delta \mathcal{E}_{j}}(0)+\mid 1\right\rangle\left\langle\left. 1\right|_{j} \otimes \hat{P}_{\Delta \mathcal{E}_{j}}(1),\right.
$$

namely, $\hat{P}_{j}$ selects those configurations such that the difference between the electric field values in neighboring links and the occupation of the site between them satisfies the Gauss law.

Now we focus on the aforementioned Hamiltonian $\hat{H}_{S}^{g}$. We show that for two basis vectors $|m, \mathcal{E}\rangle$ and $\left|m^{\prime}, \mathcal{E}^{\prime}\right\rangle$ an operator $\hat{H}_{S}^{g}$ exists such that

$$
\left\langle m, \mathcal{E}\left|\hat{H}_{S}\right| m^{\prime}, \mathcal{E}^{\prime}\right\rangle=\left\langle\mathcal{E}\left|\hat{H}_{S}^{g}\right| \mathcal{E}^{\prime}\right\rangle .
$$

In the following we explicitly compute the operator $\hat{H}_{S}^{g}$. We start by considering the mass term at the site $j$,

$$
\left\langle m, \mathcal{E}\left|\hat{\psi}_{j}^{\dagger} \hat{\psi}_{j} \otimes \mathbf{1}^{\mathcal{E}}\right| m^{\prime}, \mathcal{E}^{\prime}\right\rangle=\left\langle\mathcal{E}\left|\left(\frac{1-\hat{p}_{j}}{2}-\Delta \hat{\mathcal{E}}_{j}\right)\right| \mathcal{E}^{\prime}\right\rangle,
$$

where $\mathbf{1}^{\mathcal{E}}$ is the identity operator acting on the electric field space and we used the fact that all the basis vectors $\{|m, \mathcal{E}\rangle\}$ satisfy the Gauss law. The computation of the electric field energy is straightforward since it is diagonal in the electric field local computational basis.

The hopping term is composed of the unitary propagator, which provides the evolution of the gauge field, and the fermionic operators, which constrain the allowed transitions. In order to integrate out the matter field, we need to recast the fermionic constraints in terms of the gauge field operators only. To this purpose we observe that

$$
\begin{aligned}
\langle m, & \left.\mathcal{E}\left|\hat{\psi}_{j}^{\dagger} \hat{\mathcal{U}}_{j}^{\dagger} \hat{\psi}_{j+1}\right| m^{\prime}, \mathcal{E}^{\prime}\right\rangle \\
& =\left\langle m, \mathcal{E}\left|\hat{P} \hat{\psi}_{j}^{\dagger} \hat{\mathcal{U}}_{j}^{\dagger} \hat{\psi}_{j+1} \hat{P}\right| m^{\prime}, \mathcal{E}^{\prime}\right\rangle \\
& =\left\langle m, \mathcal{E}\left|\hat{P} \hat{P}_{j} \otimes \hat{P}_{j+1} \hat{\psi}_{j}^{\dagger} \hat{\mathcal{U}}_{j}^{\dagger} \hat{\psi}_{j+1} \hat{P}_{j} \otimes \hat{P}_{j+1} \hat{P}\right| m^{\prime}, \mathcal{E}^{\prime}\right\rangle,
\end{aligned}
$$

where we have used the definition of $\hat{P}$ as well as the fact that $\hat{P}_{j}^{2}=\hat{P}_{j} \forall j$. Discarding the overall projector $\hat{P}$ and using the definition (A2), we obtain

$$
\begin{aligned}
\langle m, \mathcal{E}|\left[| 1 \rangle \langle 1 | _ { j } \otimes \hat { P } _ { \Delta , \mathcal { E } _ { j } } ( 1 ) ] \otimes \left[|0\rangle\left\langle\left. 0\right|_{j+1} \otimes \hat{P}_{\Delta \mathcal{E}_{j+1}}(0)\right] \hat{\psi}_{j}^{\dagger} \hat{\mathcal{U}}_{j}^{\dagger}\right.\right. \\
\hat{\psi}_{j+1}\left[| 0 \rangle \langle 0 | _ { j } \otimes \hat { P } _ { \Delta \mathcal { E } _ { j } } ( 0 ) ] \otimes \left[|1\rangle\left\langle\left. 1\right|_{j+1} \otimes \hat{P}_{\Delta \mathcal{E}_{j+1}}(1)\right]\left|m^{\prime}, \mathcal{E}^{\prime}\right\rangle\right.\right. \\
=\langle m, \mathcal{E}|\left[\hat{P}_{\Delta \mathcal{E}_{j}}(1) \hat{P}_{\Delta \mathcal{E}_{j+1}}(0) \hat{\mathcal{U}}_{j}^{\dagger} \hat{P}_{\Delta \mathcal{E}_{j}}(0) \hat{P}_{\Delta \mathcal{E}_{j+1}}(1)\right] \\
\quad \otimes|1\rangle\left\langle\left. 0\right|_{j} \otimes \mid 0\right\rangle\left\langle\left. 1\right|_{j+1} \mid m^{\prime}, \mathcal{E}^{\prime}\right\rangle \\
=\left\langle\mathcal{E}\left|\left[\hat{P}_{\Delta \mathcal{E}_{j}}(1) \hat{P}_{\Delta \mathcal{E}_{j+1}}(0) \hat{\mathcal{U}}_{j}^{\dagger} \hat{P}_{\Delta \mathcal{E}_{j}}(0) \hat{P}_{\Delta \mathcal{E}_{j+1}}(1)\right]\right| \mathcal{E}^{\prime}\right\rangle \\
=\left\langle\mathcal{E}\left|\left[\hat{\mathcal{U}}_{j}^{\dagger} \hat{P}_{\Delta \mathcal{E}_{j}}(0) \hat{P}_{\Delta \mathcal{E}_{j+1}}(1)\right]\right| \mathcal{E}^{\prime}\right\rangle .
\end{aligned}
$$

The first passage is justified by observing that $\hat{\psi}_{j}^{\dagger} \hat{\psi}_{j+1}=$ $|1\rangle\left\langle\left. 0\right|_{j} \otimes \mid 0\right\rangle\left\langle\left. 1\right|_{j+1} \cdot{ }^{3}\right.$ The second passage is a consequence of

\footnotetext{
${ }^{3}$ This is true since the product of the nearest-neighbor operators $\hat{\psi}_{j}^{\dagger}$ and $\hat{\psi}_{j+1}$ is bosonic.
} 
the definition (A1), which allows us to write

$$
\left\langlem \left|\left[|1\rangle\left\langle\left. 0\right|_{j} \otimes \mid 0\right\rangle\left\langle\left. 1\right|_{j+1}\right]\left|m^{\prime}\right\rangle=1 \Longleftrightarrow\left\langle\mathcal{E}\left|\left[\hat{P}_{\Delta \mathcal{E}_{j}}(1) \hat{P}_{\Delta \mathcal{E}_{j+1}}(0) \hat{\mathcal{U}}_{j}^{\dagger} \hat{P}_{\Delta \mathcal{E}_{j}}(0) \hat{P}_{\Delta \mathcal{E}_{j+1}}(1)\right]\right| \mathcal{E}^{\prime}\right\rangle=1 .\right.\right.\right.
$$

In the last passage of Eq. (A6), since $\hat{P}_{\Delta \mathcal{E}_{j}}(1) \hat{P}_{\Delta \mathcal{E}_{j+1}}(0)=\hat{\mathcal{U}}_{j}^{\dagger} \hat{P}_{\Delta \mathcal{E}_{j}}(0) \hat{P}_{\Delta \mathcal{E}_{j+1}}(1) \hat{\mathcal{U}}_{j}$, it follows that

$$
\begin{aligned}
\hat{P}_{\Delta \mathcal{E}_{j}}(1) \hat{P}_{\Delta \mathcal{E}_{j+1}}(0) \hat{\mathcal{U}}_{j}^{\dagger} \hat{P}_{\Delta \mathcal{E}_{j}}(0) \hat{P}_{\Delta \mathcal{E}_{j+1}}(1) & =\hat{\mathcal{U}}_{j}^{\dagger} \hat{P}_{\Delta \mathcal{E}_{j}}(0) \hat{P}_{\Delta \mathcal{E}_{j+1}}(1) \hat{\mathcal{U}}_{j} \hat{\mathcal{U}}_{j}^{\dagger} \hat{P}_{\Delta \mathcal{E}_{j}}(0) \hat{P}_{\Delta \mathcal{E}_{j+1}}(1) \\
& =\hat{\mathcal{U}}_{j}^{\dagger}\left[\hat{P}_{\Delta \mathcal{E}_{j}}(0) \hat{P}_{\Delta \mathcal{E}_{j+1}}(1)\right]^{2}=\hat{\mathcal{U}}_{j}^{\dagger} \hat{P}_{\Delta \mathcal{E}_{j}}(0) \hat{P}_{\Delta \mathcal{E}_{j+1}}(1) .
\end{aligned}
$$

In conclusion, we have derived the Hamiltonian

$$
\hat{H}_{S}^{g}=-t \sum_{j} \hat{\mathcal{U}}_{j}^{\dagger} \hat{P}_{\Delta \mathcal{E}_{j}}(0) \hat{P}_{\Delta \mathcal{E}_{j+1}}(1)+\text { H.c. }+m \sum_{j}\left(\frac{1-p_{j}}{2}-\Delta \hat{\mathcal{E}}_{j}\right)+g^{2} \sum_{j} \hat{\mathcal{E}}_{j}^{2} .
$$

\section{APPENDIX B: SECOND-ORDER DERIVATION OF THE HAMILTONIAN $\hat{\boldsymbol{H}}_{r}^{\Phi}$}

In this Appendix we show how to map the Rydberg Hamiltonian to the $\mathbf{Z}_{3}$ Hamiltonian (3) by using second-order perturbation theory. We start by considering the single-link case and then we extend the result to the entire chain. The Hamiltonian $\hat{H}_{r}$ [Eq. (4)], in terms of single-link 3-atom states $\{|r g g\rangle,|g r g\rangle,|g g r\rangle,|g g g\rangle,|g r r\rangle,|r g r\rangle,|r r g\rangle,|r r r\rangle\}$, reduces to the $8 \times 8$ matrix

$$
\hat{H}_{r, s}=\left(\begin{array}{ccc||c|ccc|c}
\delta_{0} & 0 & 0 & \Omega & 0 & \Omega & \Omega & 0 \\
0 & \delta_{1} & 0 & \Omega & \Omega & 0 & \Omega & 0 \\
0 & 0 & \delta_{2} & \Omega & \Omega & \Omega & 0 & 0 \\
\hline \hline \Omega & \Omega & \Omega & \Delta & 0 & 0 & 0 & 0 \\
\hline 0 & \Omega & \Omega & 0 & V_{\ell}-\Delta & 0 & 0 & \Omega \\
\Omega & 0 & \Omega & 0 & 0 & V_{\ell}-\Delta & 0 & \Omega \\
\Omega & \Omega & 0 & 0 & 0 & 0 & V_{\ell}-\Delta & \Omega \\
\hline 0 & 0 & 0 & 0 & \Omega & \Omega & \Omega & 2\left(V_{\ell}-\Delta\right)
\end{array}\right) \equiv\left(\begin{array}{c}
\hat{H}_{r, s}^{P P} \| \hat{H}_{r, s}^{P N} \\
\hline \hline \hat{H}_{r, s}^{N P}
\end{array} \hat{H}_{r, s}^{N N}\right) .
$$

The first three states contain only one excited atom and they form the set $\Sigma=\{|\vartheta\rangle\}_{0 \leqslant \vartheta \leqslant 2}$ defined in the main text (link indices are omitted here). We have applied a shift of the energy equal to $\Delta$, so the energy of the states in $\Sigma$ is $E_{s} \sim \delta_{\vartheta}$. We call $\mathcal{H}_{s}^{P}$ the subspace spanned by $\Sigma$ and $\mathcal{H}_{s}^{N}$ the complementary one such that $\mathcal{H}_{s}=\mathcal{H}_{s}^{N} \oplus \mathcal{H}_{s}^{P}$. We define the projectors $\hat{P}_{s}$ and $\hat{N}_{s}=\mathbf{1}-\hat{P}_{s}$ such that

$$
\begin{aligned}
& \hat{H}_{r, s}^{P P}=\hat{P}_{s} \hat{H}_{r, s} \hat{P}_{s}, \\
& \hat{H}_{r, s}^{N N}=\hat{N}_{s} \hat{H}_{r, s} \hat{N}_{s}, \\
& \hat{H}_{r, s}^{P N}=\hat{P}_{s} \hat{H}_{r, s} \hat{N}_{s}=\left(\hat{H}_{r, s}^{N P}\right)^{\dagger} .
\end{aligned}
$$

They correspond, respectively, to the top left, bottom right, and off-diagonal parts of $\hat{H}_{r, s}$ delimited by double lines in Eq. (B1). The effective Hamiltonian relative to the subspace $\mathcal{H}_{s}^{P}$ can be derived by assuming that there exists a set of eigenstates of $\hat{H}_{r, s}$ whose energies are perturbations of the spectrum of $\hat{H}_{r, s}^{P P}$. Let us consider an eigenstate $|\Psi\rangle$ whose energy satisfies $E_{s} \sim \delta_{\vartheta} \ll V_{\ell}-\Delta, \Delta$ and define $\left|\Psi_{P}\right\rangle=\hat{P}_{S}|\Psi\rangle$ and $\left|\Psi_{N}\right\rangle=\hat{N}_{S}|\Psi\rangle$; the eigenvalue equation can be written as

$$
E_{s}\left[\begin{array}{l}
\left|\Psi_{P}\right\rangle \\
\left|\Psi_{N}\right\rangle
\end{array}\right]=\left[\begin{array}{ll}
\hat{H}_{r, s}^{P P} & \hat{H}_{r, s}^{P N} \\
\hat{H}_{r, s}^{N P} & \hat{H}_{r, s}^{N N}
\end{array}\right]\left[\begin{array}{c}
\left|\Psi_{P}\right\rangle \\
\left|\Psi_{N}\right\rangle
\end{array}\right]
$$

from which it emerges that $\left|\Psi_{P}\right\rangle$ obeys the equation

$$
E_{s}\left|\Psi_{P}\right\rangle=\left[\hat{H}_{r, s}^{P P}+\hat{H}_{r, s}^{P N} \frac{1}{E_{s}-\hat{H}_{r, s}^{N N}} \hat{H}_{r, s}^{N P}\right]\left|\Psi_{P}\right\rangle=\hat{\tilde{H}}_{r, s}^{P P}\left|\Psi_{P}\right\rangle .
$$

We compute the matrix elements of $\hat{\tilde{H}}_{r, s}^{P P}$ in the basis of the states $\{|r g g\rangle,|g r g\rangle,|g g r\rangle\}$. We consider its action on the state $|r g g\rangle$ :

$$
\begin{aligned}
\hat{\tilde{H}}_{r, s}^{P P}|r g g\rangle= & \delta_{0}|r g g\rangle+\Omega \hat{H}_{r, s}^{P N} \frac{1}{E_{s}-\hat{H}_{r, s}^{N N}}(|g g g\rangle \\
& +|r r g\rangle+|r g r\rangle) .
\end{aligned}
$$

We approximate the operator $\hat{H}_{r, s}^{N N}$ with its diagonal contribution, since $\Omega$ is much smaller than $\Delta, V_{\ell}-\Delta$. In Eq. (B5) we use $1 /\left(E_{s}-\hat{H}_{r, s}^{N N}\right) \simeq-\left(\hat{H}_{r, s}^{N N}\right)_{\text {diag }}^{-1}$, where we have neglected $E_{s} \ll \Delta, V_{\ell}-\Delta$, and therefore we get

$$
\begin{aligned}
\hat{\tilde{H}}_{r, s}^{P P}|r g g\rangle \\
\simeq \delta_{1}|r g g\rangle-\Omega \hat{H}_{r, s}^{P N}\left(\hat{H}_{r, s}^{N N}\right)_{\mathrm{diag}}^{-1}(|g g g\rangle+|r r g\rangle+|r g r\rangle) \\
=\delta_{1}|r g g\rangle-\Omega \hat{H}_{r, s}^{P N}\left[\frac{1}{\Delta}|g g g\rangle+\frac{1}{V_{\ell}-\Delta}(|r r g\rangle+|r g r\rangle)\right] \\
=\left[\delta_{1}-\Omega^{2}\left(\frac{1}{\Delta}+\frac{2}{V_{\ell}-\Delta}\right)\right]|r g g\rangle \\
+\Omega^{2}\left(\frac{1}{\Delta}+\frac{1}{V_{\ell}-\Delta}\right)|g r g\rangle+\Omega^{2}\left(\frac{1}{\Delta}+\frac{1}{V_{\ell}-\Delta}\right)|g g r\rangle .
\end{aligned}
$$

By repeating the same procedure for the states $|g r g\rangle$ and $|g g r\rangle$ we obtain the effective Hamiltonian

$$
\hat{H}_{r, s}^{\Phi}=-t_{s} \sum_{\vartheta \neq \vartheta^{\prime}}|\vartheta\rangle\left\langle\vartheta^{\prime}\left|+\sum_{\vartheta} \delta_{\vartheta}\right| \vartheta\right\rangle\langle\vartheta|,
$$




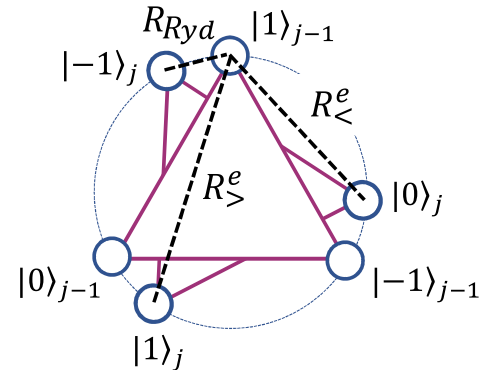

FIG. 5. Mass implementation. In the case with $m \neq 0$ the positions of the atoms belonging to even links are rotated counterclockwise by an angle $\phi$. The correspondent value of the mass $m=V_{c}^{e}-V_{v}^{e}$ is implemented.

with $t_{s}=\Omega^{2}\left(\frac{1}{V-\Delta}+\frac{1}{\Delta}\right)$, where we are neglecting the overall constant $-\Omega^{2}\left(\frac{2}{V-\Delta}+\frac{1}{\Delta}\right)$.

When we consider a chain of $L$ links we must take into account the interlink energies $V$ and $V_{d}$. In fact, the interactions between nearest neighbors modify the local Hamiltonian $\hat{H}_{r, s}^{N N}$. Transitions between different gauge-invariant states $|\Psi\rangle$ and $\left|\Psi^{\prime}\right\rangle$ are mediated by local link changes $|\vartheta\rangle_{j} \rightarrow\left|\vartheta^{\prime}\right\rangle_{j}$.

For example, let us suppose that the transition $|\Psi\rangle \rightarrow\left|\Psi^{\prime}\right\rangle$ corresponds to only changing the local state $|r g g\rangle_{j}$ to the state $|g r g\rangle_{j}$. This transition is now mediated by the doubly excited local state $|r r g\rangle_{j}$ and by the local state $|g g g\rangle_{j}$ with no excitations. Due to the interlink interaction, the transition $|r g g\rangle_{j} \rightarrow|r r g\rangle_{j}$ induces a local change in the energy $V_{\ell}+$ $2 V-\Delta$. Analogously, the transition $|r g g\rangle_{j} \rightarrow|g g g\rangle_{j}$ leads to an energy change $\Delta-2 V$. As a consequence, the allowed transition $|r g g\rangle_{j} \rightarrow|g r g\rangle_{j}$ is a second-order process with rate $t=\Omega^{2}\left(\frac{1}{V_{\ell}+2 V-\Delta}+\frac{1}{\Delta-2 V}\right)$.

So far, we only consider the transition between gauge-invariant many-body states. However, the Hamiltonian $\hat{H}_{r}$ may allow local transitions such as $|r g g\rangle_{j}|g g r\rangle_{j+1} \rightarrow|r g r\rangle_{j}|g g r\rangle_{j+1} \rightarrow|g g r\rangle_{j}|g g r\rangle_{j+1}$ with two excited atoms at distance $d$. Such a transition is suppressed by an energy penalty $V_{d}$ and breaks the gauge invariance. As long as $V_{d} \gg V, \Omega$, these second-order processes can be neglected. In conclusion, we have shown how $\hat{H}_{r}$ reduces to $\hat{H}_{r}^{\Phi}$ up to second-order corrections in $\Omega$.

\section{APPENDIX C: MASS-TERM IMPLEMENTATION}

In this Appendix we describe how to implement a nonzero mass term in the Hamiltonian $\hat{H}_{r}$. Let us recall that, in the $\mathbf{Z}_{3}$ model, even matter sites can contain a zero or negative charge corresponding, respectively, to a zero or $m>0$ mass energy. If we consider the electric field Hamiltonian $\hat{H}$, given an even site $j$, the energies of the two local states $|E\rangle_{j-1} \otimes|(E-1) \bmod 3\rangle_{j}$ and $|E\rangle_{j-1} \otimes|E\rangle_{j}$ differ by $m$. Similar considerations apply for odd sites.

In order to achieve this condition, we tilt the triangular structures relative to the even links by a small angle $\phi$ in a counterclockwise way, as shown in Fig. 5. A rotation in the plane perpendicular to the lattice axis makes the distances between the site corresponding to $E_{j-1}=-1$ and the sites
$E_{j}=-1$ and $E_{j}=0$ different. In this way, different interaction strengths are engineered and thus the energy difference between the vacuum and the charged configuration can be implemented. We define the following characteristic interlink distances:

$$
\begin{aligned}
R_{R y d} & =\left[d^{2}+4 \ell^{2} \sin ^{2}(\phi / 2)\right]^{1 / 2}, \\
R_{>} & =\left[d^{2}+4 / 3 \ell^{2} \sin ^{2}(\pi / 3+\phi / 2)\right]^{1 / 2}, \\
R_{<} & =\left[d^{2}+4 / 3 \ell^{2} \sin ^{2}(\pi / 3-\phi / 2)\right]^{1 / 2} .
\end{aligned}
$$

Here $R_{R y d}$ is the smallest distance, corresponding to forbidden configurations, while $R_{\lessgtr}$ correspond to the allowed ones.

The energies corresponding to charged and vacuum configurations are $V_{c}^{e}=c_{6} / R_{<}^{6}$ and $V_{v}^{e}=c_{6} / R_{>}^{6}$, respectively. Analogously, we define the same energies for odd matter sites, namely, $V_{c}^{o}=c_{6} / R_{<}^{6}$ and $V_{v}^{o}=c_{6} / R_{>}^{6}$.

Staggering is implemented by a further lattice deformation. Indeed, the energy of the vacuum configuration for an even matter site must be equal to the energy of the charged configuration for an odd matter site, i.e., $V_{v}^{e}=V_{c}^{o}$. In order to achieve the above statement we change the interlink distance of the chain by a small amount $\varepsilon$ such that $d^{o}=d+\varepsilon$ and $d^{e}=d-\varepsilon$ relative to odd and even sites, respectively. We choose a value of $\phi$ and thereafter choose a value of $\varepsilon$ to satisfy the condition $V_{v}^{e}=V_{c}^{o}$. As a result we get $m=V_{c}^{e}-V_{v}^{e} \simeq V_{c}^{o}-V_{v}^{o}$. For example, with the parameters used in the main text, by applying a rotation $\phi=0.05 \mathrm{rad}$ we obtain $t=0.667 \mathrm{MHz}, m_{e^{-}}=V_{c}^{e}-V_{v}^{e}=$ $0.385 \mathrm{MHz}$, and $m_{e^{+}}=V_{c}^{o}-V_{v}^{o}=0.356 \mathrm{MHz}$. We can simulate therefore a mass $m=\left(m_{e^{+}}+m_{e^{-}}\right) / 2$ with a relative error $\sim 4 \%$.

\section{APPENDIX D: DETAILS OF THE TEBD MATRIX PRODUCT STATES NUMERICAL SIMULATION}

The dynamics of the Rydberg-atom quantum simulator has been numerically simulated by using the Hamiltonian $\hat{H}_{r}$ defined in Eq. (4). We considered only interactions between nearest-neighbor links. We used the matrix product state representation of the many-body state. We took the link as local subspace: Since it is composed of three atoms which can be in a Rydberg or in the internal ground state, its Hilbert space dimension is $2^{3}=8$. The auxiliary dimension was set to 128 and we checked the convergence of the dynamics by repeating the same simulations with larger bond dimension equal to 256 . Note that the dynamics of each link is mostly constrained in the three-dimensional subspace spanned by $\Sigma_{j}$, allowing accurate results with a relatively small bond dimension.

The dynamics has been computed by using the timeevolving block decimation algorithm with second-order Suzuki-Trotter decomposition of the evolution operator, with time step $d t=0.01(2 \pi)^{-1} \mu \mathrm{s}$. Local and interaction parameters have been chose such that $\{\Delta=$ $\left.27 \mathrm{MHz}, V_{\ell}=149.414 \mathrm{MHz}, \Omega=3 \mathrm{MHz}, \delta_{\vartheta}=g^{2}\right\}$ and $\left\{V=6.186 \mathrm{MHz}, V_{d}=22.109 \mathrm{MHz}\right\}$. 
[1] A. W. Glaetzle, M. Dalmonte, R. Nath, I. Rousochatzakis, R. Moessner, and P. Zoller, Phys. Rev. X 4, 041037 (2014).

[2] H. Labuhn, D. Barredo, S. Ravets, S. de Léséleuc, T. Macrì, T. Lahaye, and A. Browaeys, Nature (London) 534, 667 (2016).

[3] H. Bernien, S. Schwartz, A. Keesling, H. Levine, A. Omran, H. Pichler, S. Choi, A. S. Zibrov, M. Endres, M. Greiner, V. Vuletic, and M. D. Lukin, Nature (London) 551, 579 (2017).

[4] V. Lienhard, S. de Léséleuc, D. Barredo, T. Lahaye, A. Browaeys, M. Schuler, L.-P. Henry, and A. M. Läuchli, Phys. Rev. X 8, 021070 (2018).

[5] H. Weimer, M. Müller, I. Lesanovsky, P. Zoller, and H. P. Büchler, Nat. Phys. 6, 382 (2010).

[6] A. Omran, H. Levine, A. Keesling, G. Semeghini, T. T. Wang, S. Ebadi, H. Bernien, A. S. Zibrov, H. Pichler, S. Choi et al., Science 365, 570 (2019).

[7] T. M. Wintermantel, Y. Wang, G. Lochead, S. Shevate, G. K. Brennen, and S. Whitlock, Phys. Rev. Lett. 124, 070503 (2020).

[8] S. de Léséleuc, D. Barredo, V. Lienhard, A. Browaeys, and T. Lahaye, Phys. Rev. A 97, 053803 (2018).

[9] I. Buluta, S. Ashhab, and F. Nori, Rep. Prog. Phys. 74, 104401 (2011).

[10] A. Gaëtan, Y. Miroshnychenko, T. Wilk, A. Chotia, M. Viteau, D. Comparat, P. Pillet, A. Browaeys, and P. Grangier, Nat. Phys. 5, 115 (2009).

[11] H. Levine, A. Keesling, A. Omran, H. Bernien, S. Schwartz, A. S. Zibrov, M. Endres, M. Greiner, V. Vuletić, and M. D. Lukin, Phys. Rev. Lett. 121, 123603 (2018).

[12] D. Barredo, S. Ravets, H. Labuhn, L. Béguin, A. Vernier, F. Nogrette, T. Lahaye, and A. Browaeys, Phys. Rev. Lett. 112, 183002 (2014).

[13] D. Barredo, V. Lienhard, S. de Léséleuc, T. Lahaye, and A. Browaeys, Nature (London) 561, 79 (2018).

[14] F. M. Surace, P. P. Mazza, G. Giudici, A. Lerose, A. Gambassi, and M. Dalmonte, arXiv:1902.09551.

[15] J. Preskill, arXiv:1811.10085.

[16] U.-J. Wiese, Ann. Phys. (Berlin) 525, 777 (2013).

[17] M. Dalmonte and S. Montangero, Contemp. Phys. 57, 388 (2016).

[18] D. Banerjee, M. Dalmonte, M. Müller, E. Rico, P. Stebler, U.-J. Wiese, and P. Zoller, Phys. Rev. Lett. 109, 175302 (2012).

[19] K. Stannigel, P. Hauke, D. Marcos, M. Hafezi, S. Diehl, M. Dalmonte, and P. Zoller, Phys. Rev. Lett. 112, 120406 (2014).

[20] E. Zohar, J. I. Cirac, and B. Reznik, Phys. Rev. Lett. 109, 125302 (2012).

[21] E. Zohar, J. I. Cirac, and B. Reznik, Phys. Rev. Lett. 110, 055302 (2013).

[22] E. Zohar, J. I. Cirac, and B. Reznik, Phys. Rev. Lett. 110, 125304 (2013).

[23] T. V. Zache, F. Hebenstreit, F. Jendrzejewski, M. K. Oberthaler, J. Berges, and P. Hauke, Quantum Sci. Technol. 3, 034010 (2018).

[24] L. Tagliacozzo, A. Celi, P. Orland, M. W. Mitchell, and M. Lewenstein, Nat. Commun. 4, 2615 (2013).

[25] K. Kasamatsu, I. Ichinose, and T. Matsui, Phys. Rev. Lett. 111, 115303 (2013).

[26] Y. Kuno, S. Sakane, K. Kasamatsu, I. Ichinose, and T. Matsui, Phys. Rev. D 95, 094507 (2017).
[27] Y. Kuno, S. Sakane, K. Kasamatsu, I. Ichinose, and T. Matsui, Phys. Rev. A 94, 063641 (2016).

[28] J. Park, Y. Kuno, and I. Ichinose, Phys. Rev. A 100, 013629 (2019).

[29] E. A. Martinez, C. A. Muschik, P. Schindler, D. Nigg, A Erhard, M. Heyl, P. Hauke, M. Dalmonte, T. Monz, P. Zoller, and R. Blatt, Nature (London) 534, 516 (2016).

[30] A. Mil, T. V. Zache, A. Hegde, A. Xia, R. P. Bhatt, M. K. Oberthaler, P. Hauke, J. Berges, and F. Jendrzejewski, Science 367, 1128 (2020).

[31] C. Schweizer, F. Grusdt, M. Berngruber, L. Barbiero, E. Demler, N. Goldman, I. Bloch, and M. Aidelsburger, Nat. Phys. 15, 1168 (2019).

[32] C. Kokail, C. Maier, R. van Bijnen, T. Brydges, M. K. Joshi, P. Jurcevic, C. A. Muschik, P. Silvi, R. Blatt, C. F. Roos, and P. Zoller, Nature (London) 569, 355 (2019).

[33] N. Klco, E. F. Dumitrescu, A. J. McCaskey, T. D. Morris, R. C. Pooser, M. Sanz, E. Solano, P. Lougovski, and M. J. Savage, Phys. Rev. A 98, 032331 (2018).

[34] J. C. Pinto Barros, M. Dalmonte, and A. Trombettoni, Phys. Rev. D 100, 036009 (2019).

[35] B. Buyens, J. Haegeman, H. Verschelde, F. Verstraete, and K. Van Acoleyen, Phys. Rev. X 6, 041040 (2016).

[36] T. Pichler, M. Dalmonte, E. Rico, P. Zoller, and S. Montangero, Phys. Rev. X 6, 011023 (2016).

[37] M. Pepe and U.-J. Wiese, Phys. Rev. Lett. 102, 191601 (2009).

[38] A. Celi, B. Vermersch, O. Viyuela, H. Pichler, M. D. Lukin, and P. Zoller, arXiv:1907.03311.

[39] J. Kogut and L. Susskind, Phys. Rev. D 11, 395 (1975).

[40] I. Montvay and G. Munster, Quantum Fields on a Lattice (Cambridge University Press, Cambridge, 1997).

[41] E. Zohar and J. I. Cirac, Phys. Rev. D 99, 114511 (2019).

[42] E. Zohar and J. I. Cirac, Phys. Rev. B 98, 075119 (2018).

[43] S. Notarnicola, E. Ercolessi, P. Facchi, G. Marmo, S. Pascazio, and F. V. Pepe, J. Phys. A: Math. Theor. 48, 30FT01 (2015).

[44] E. Ercolessi, P. Facchi, G. Magnifico, S. Pascazio, and F. V. Pepe, Phys. Rev. D 98, 074503 (2018).

[45] M. A. Norcia, A. W. Young, and A. M. Kaufman, Phys. Rev. X 8, 041054 (2018).

[46] A. Cooper, J. P. Covey, I. S. Madjarov, S. G. Porsev, M. S. Safronova, and M. Endres, Phys. Rev. X 8, 041055 (2018).

[47] P. Schauß, M. Cheneau, M. Endres, T. Fukuhara, S. Hild, A. Omran, T. Pohl, C. Gross, S. Kuhr, and I. Bloch, Nature (London) 491, 87 (2012).

[48] G. Vidal, Phys. Rev. Lett. 93, 040502 (2004).

[49] U. Schollwöck, Ann. Phys. (NY) 326, 96 (2011).

[50] T. V. Zache, N. Mueller, J. T. Schneider, F. Jendrzejewski, J. Berges, and P. Hauke, Phys. Rev. Lett. 122, 050403 (2019).

[51] G. Magnifico, D. Vodola, E. Ercolessi, S. P. Kumar, M. Müller, and A. Bermudez, Phys. Rev. D 99, 014503 (2019).

[52] G. Magnifico, D. Vodola, E. Ercolessi, S. Kumar, M. Müller, and A. Bermudez, Phys. Rev. B 100, 115152 (2019).

[53] C. Fan, D. Rossini, H.-X. Zhang, J.-H. Wu, M. Artoni, and G. La Rocca, Phys. Rev. A 101, 013417 (2020).

[54] F. M. Surace, A. Russomanno, M. Dalmonte, A. Silva, R. Fazio, and F. Iemini, Phys. Rev. B 99, 104303 (2019). 